\title{
A ROOT Tool for 3D Event Visualization in ATLAS Calorimeter
}

\section{L.M. de Andrade Filho*}

Signal Processing Lab/Federal University of Rio de Janeiro

E-mail: lucianof@lps.ufrj.br

\section{J.M. de Seixas}

Signal Processing Lab/Federal University of Rio de Janeiro

E-mail: seixas@lps.ufrj.br

The ATLAS (A Toroidal LHC ApparatuS) detector is being tested with cosmic rays before LHC (Large Hadron Collider) starts its full operation by the midle of the year 2008. For the ongoing commissioning phase, it is necessary to develop specific tools that can perform efficient cosmic ray data analysis. An important issue for final analysis is to provide a way to visualize cosmic muon tracks and the corresponding activated cells in the detector, so that one can check visually the coherence of the reconstructed data and seek for potential problems. This work presents a 3D visualization tool for cosmic muon track visualization based on activated cells in the highly segmented ATLAS calorimeter system. This tool was developed in the ROOT framework, which allows a smooth integration between specifics analyses from ATLAS community and the visualization tool. This tool uses the ROOT embedded geometry package to create the ATLAS calorimeter, cell by cell, and provides routines to fill calorimeter cells with the reconstructed data information. Energy thresholds can be set by the user so that only relevant information is displayed. The tool structure and some applicationand with reconstructed data are presented.

XI International Workshop on Advanced Computing and Analysis Techniques in Physics Research April 23-27 2007

Amsterdam, the Netherlands

\footnotetext{
* Speaker.
} 


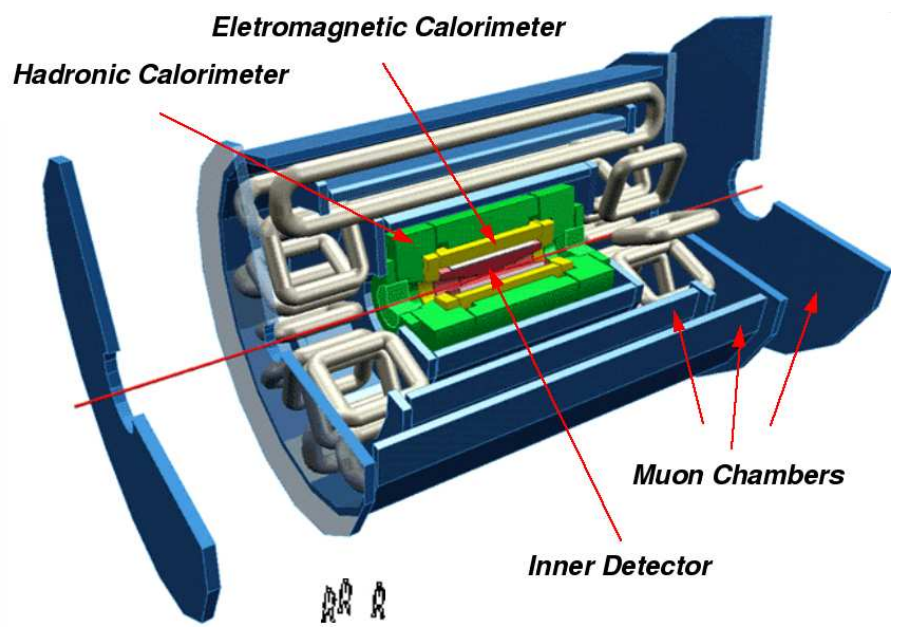

Figure 1: ATLAS detector overview.

\section{Introduction}

ATLAS [1] is a particle physics experiment that will explore the fundamental nature of matter and the basic forces that shape our universe. Starting in mid-2008, the ATLAS detector will search for new discoveries in the head-on collisions of protons of extraordinarily high energy. The detector is a cylinder with a total length of $42 \mathrm{~m}$ and a radius of $11 \mathrm{~m}$ (see Figure 1).

The Inner Detector [2] starts a few centimeters from the proton beam axis, extending to a radius of $1.2 \mathrm{~m}$, and is $7 \mathrm{~m}$ in length along the beam pipe. Its basic function is to track charged particles by detecting their interaction with material at discrete points, revealing detailed information about the type of particle and its momentum. A magnetic field surrounding the entire inner detector causes charged particles to curve; the direction of the curve reveals a particle's charge and the degree of curvature reveals its momentum.

The calorimeters are situated outside the solenoidal magnet that surrounds the inner detector. Their purpose is to measure the energy from particles by absorbing it. The calorimeter system comprises two sections [3]: an inner electromagnetic calorimeter and an outer hadronic calorimeter. Both are sampling calorimeters; that is, they absorb energy in high-density metal and periodically sample the shape of the resulting particle shower, inferring the energy of the original particle from this measurement.

The electromagnetic (EM) calorimeter [4] absorbs energy from particles that interact electromagnetically, which include charged particles and photons. It has high precision, both in the amount of energy absorbed and in the precise location of the energy deposited. The angle between the particle's trajectory and the detector's beam axis (or more precisely the pseudorapidity $\eta$ ) and its angle within the perpendicular plane are both measured to within roughly 0.025 radians. The energy-absorbing materials are lead and stainless steel, with liquid argon as the sampling material, and a cryostat is required around the EM calorimeter to keep it sufficiently cool.

The hadron calorimeter absorbs energy from particles that pass through the EM calorimeter, but do interact via the strong force; these particles are primarily hadrons. It is less precise, both in energy magnitude and in the localization (within about 0.1 radians only) [5]. The energy-absorbing 


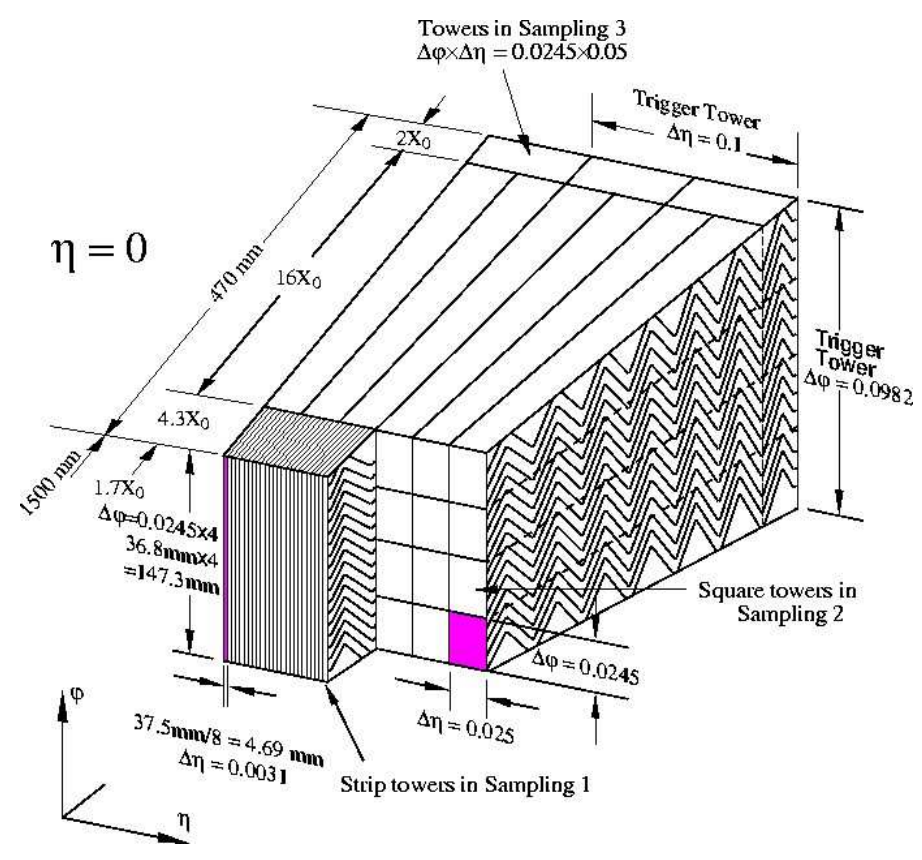

Figure 2: ATLAS electromagnetic calorimeter segmentation (barrel part).

material is steel, with scintillating tiles that sample the energy deposited. Many of the features of the calorimeter are chosen for their cost-effectiveness; the instrument is large and comprises a huge amount of construction material: the main part of the calorimeter - the tile calorimeter - is $8 \mathrm{~m}$ in diameter and covers $12 \mathrm{~m}$ along the beam axis. The far-forward sections of the hadronic calorimeter are contained within the EM calorimeter's cryostat, and use liquid argon as it does.

The muon spectrometer is an extremely large tracking system, extending from a radius of 4.25 $m$ around the calorimeters out to the full radius of the detector $(11 \mathrm{~m})$ [6]. Its tremendous size is required to accurately measure the momentum of muons, which penetrate other elements of the detector; the effort is vital because one or more muons are a key element of a number of interesting physical processes, and because the total energy of particles in an event could not be measured accurately if they were ignored. It functions similarly to the inner detector, with muons curving so that their momentum can be measured, albeit with a different magnetic field configuration, lower spatial precision, and a much larger volume. It also serves the function of simply identifying muons - very few particles of other types are expected to pass through the calorimeters and subsequently leave signals in the muon spectrometer. It has roughly one million readout channels, and its layers of detectors have a total area of $12,000 \mathrm{~m}^{2}$.

\section{Calorimeter Segmentation}

Figure 2 shows the segmentation for the barrel part of the electromagnetic calorimeter. It has three regions with different granularities in a total of around 100,000 readout channels. The dimensions are given in terms of ATLAS coordinates $\eta \times \phi$ [7].

1. Sampling 1 (S1 or Strips region) has a very fine granularity and a depth of 4.3 radiation lengths; this region is used for $\lambda / \pi^{0}$ and $e / \lambda$ separation; 

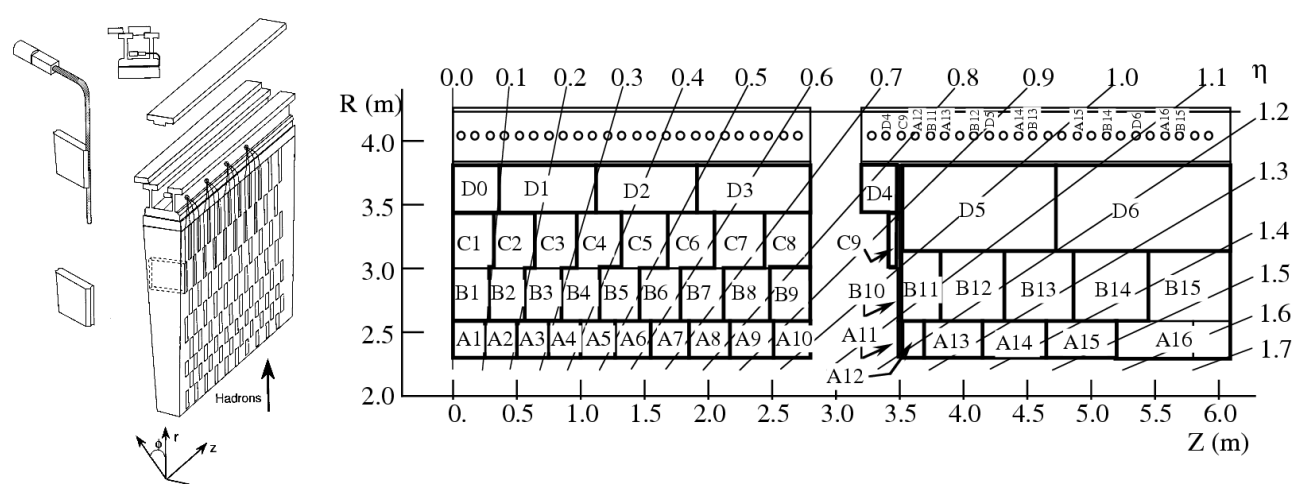

Figure 3: ATLAS hadronic calorimeter segmentation (TileCal part).

2. Sampling 2 (S2 or Middle region) has a granularity of $0.025 \times 0.0245$ and a depth of 16 radiation lengths; most of the energy of electromagnetic showers is deposited here;

3. Sampling 3 (S3 or Back region) has a coarse granularity $0.05 \times 0.0245$ and a depth of 2 radiation lengths; this region is used to measure the tail of high energy showers, which extend past $\mathrm{S} 2$; this region is also used to separate between hadronic and electromagnetic showers, since the former deposit a larger amount of energy in S3.

The Hadronic Tile Calorimeter is composed of three cylindrical parts: one barrel sub-detector, covering the rapidity range $|\eta|<1.0$ and two extended barrel wheels, covering the rapidity range $0.8<|\eta|<1.7$ on either side of the barrel. All the sub-detectors are located around the beam axis; the barrel and the two extended barrels are separated by a gap of $600 \mathrm{~mm}$ to provide space for liquid argon distribution pipes. Each of the sub-detectors is composed of 64 azimuthal wedgeshaped module. Figure 3 shows the tile calorimeter segmentation. On the left, the connection between a tile and a PMT via optical fibres is shown. In this subdetector each tile is coupled to two PMTs for redundancy. The scintillating tiles slide into the gaps created in the steel matrix of the module (right).

\section{Trigger System}

In order to identify interesting and rare physics phenomena in the bulk of the data, the ATLAS detector implements a three-level trigger (Figure 4). The task of the trigger [8] is to classify events according to landmark properties and to store those that comply with a list of given descriptions. This list is currently referred to as the trigger menu. The three trigger levels have different requirements and different implementation strategies:

1. Level1 The first level (LVL1) trigger is optimized for maximum processing speed. It processes a subset of the available data: Calorimeters are read out only with coarse granularity, while fast Muon Trigger detectors are read out with full granularity. To further reduce processing time, the trigger is implemented with dedicated electronics, located as close to the detector as possible. 


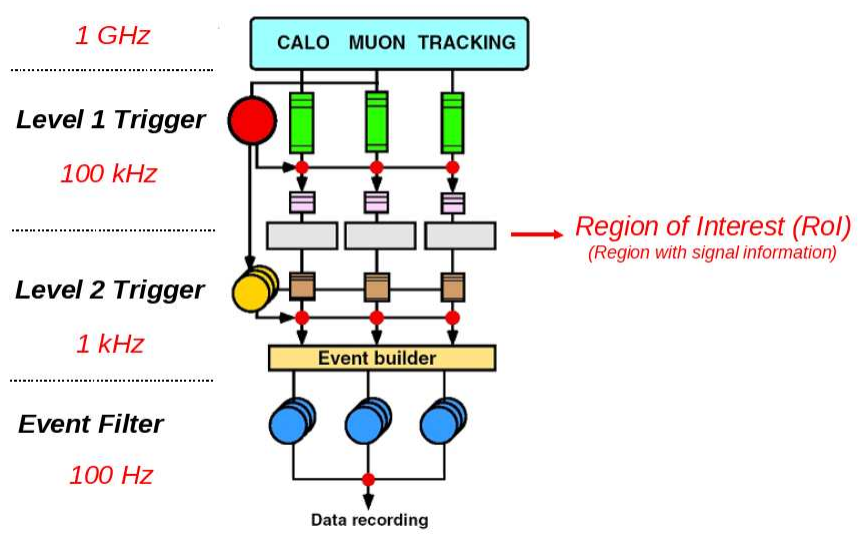

Figure 4: The ATLAS three-level trigger.

2. Level2 The second level (LVL2) trigger receives input from LVL1 for accepted events. This input consists of Regions of Interest (RoIs); the RoIs represent signal areas in the subdetectors that are recognized by the first level trigger as containing a trigger item. The design input rate from LVL1 is $100 \mathrm{kHz}$, which limits the average processing time for LVL2 algorithms - running on multiple PCs - to less than $10 \mathrm{~ms}$.

3. Event Filter The Event Filter receives LVL2 decisions with an input rate of $3.5 \mathrm{kHz}$, then proceeds to read out the full event data from all the sub-detectors. The processing time is expected to be on average 1 second per event, and the result is stored in a permanent repository. The Event Filter is implemented on a large farm of PCs, and the output rate for reconstructed data is $200 \mathrm{~Hz}$.

\section{Commissioning with cosmic rays}

The goal of the calorimeter commissioning with cosmic rays [9] is the validation of the performance of the calibrated detector, ensuring the readiness for LHC turn-on. In order to acquire cosmic data, the trigger system is bypassed and a coincidence board assembled for this proposal [10] is used. Figure 5 shows how the setup works. If any cell in the top and in the bottom parts of the hadronic calomimeter are excited at the same time, a trigger signal is generates and the data for the both hadronic and electromagnetic calorimeters are recorded.

Cosmic ray muons can be used to check various detector parameters. While the cell by cell statistics might not be sufficient, it will be possible to sum similar cells (along the $\phi$ direction) and perform physics signal shape study, as well as amplitude and time inter-calibrations. The problem channels, such as HV different from nominal voltage, dead readout channels etc, can be identified, and proper treatment of these channels can be investigated. To help in these tasks, a 3D visualization for the excited calorimeters cells has been an easy and intuitive way to identify problems.

This work presents a tool for 3D visualization of the calorimeter cells with features that allow to check the coherency of the online software algorithms and support the commissioning phase 


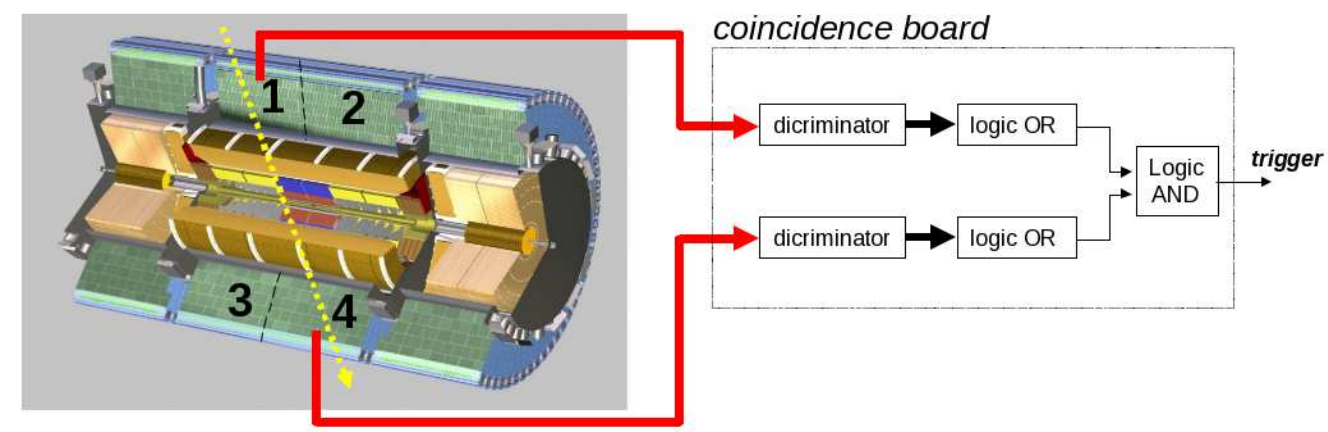

Figure 5: Triggering cosmic rays with TileCal.

with cosmic rays. This tool was developed using ROOT framework [11] and its embedded geometry package [12]. Since the framework for data reconstruction in ATLAS, the Athena [13], generates files specific to be loaded in ROOT, the integration between reconstructed data and this visualization tool can be done smoothly.

\section{The Geometry Tool}

The proposed tool comes in two different versions: a TileCal specific tool (the TileCosmicView) and a combined TileCal + LArg tool (the CaloGeoView). The first one is used to load specific TileCal reconstructed ntuples where more detailed information from this subdetector, as pulse-shape and energy to each PMT, can be accessed, increasing the commissioning potential for the hadronic calorimeter. The CaloGeoView uses Athena aware ntuples. The difference between these two ntuples is that the second one has information about the energy deposited in each cell for both calorimeters, but the pulse-shape and the energy information to each TileCal PMT are not present.

\subsection{The TileCosmicView}

Figure 6 shows the graphical interface for the TileCosmicView. Two main windows can be identified. The left most window (ROOT's GL viewer) is the standard viewer for 3D objects in ROOT. A recently acquired cosmic event is showed in the center. In this window we can identify the TileCal outline, showed in a transparent gray color, some cells activated by the cosmic muon and its corresponding track. The right window is the Graphical User Interface (GUI), which has controls to open, access and visualize the informations within the reconstructed ntuples. The main features of this tool are:

- Fast response - the commands are executed quickly taking only some miliseconds to be performed.

- Color Palette adjustment - the colors of the activated cells depend on the energy deposited in the cell. A color palette from yellow to dark red is used (hot palette) with yellow assigned to energy $=0$. The energy assigned to the dark red color is chosen by the user. 


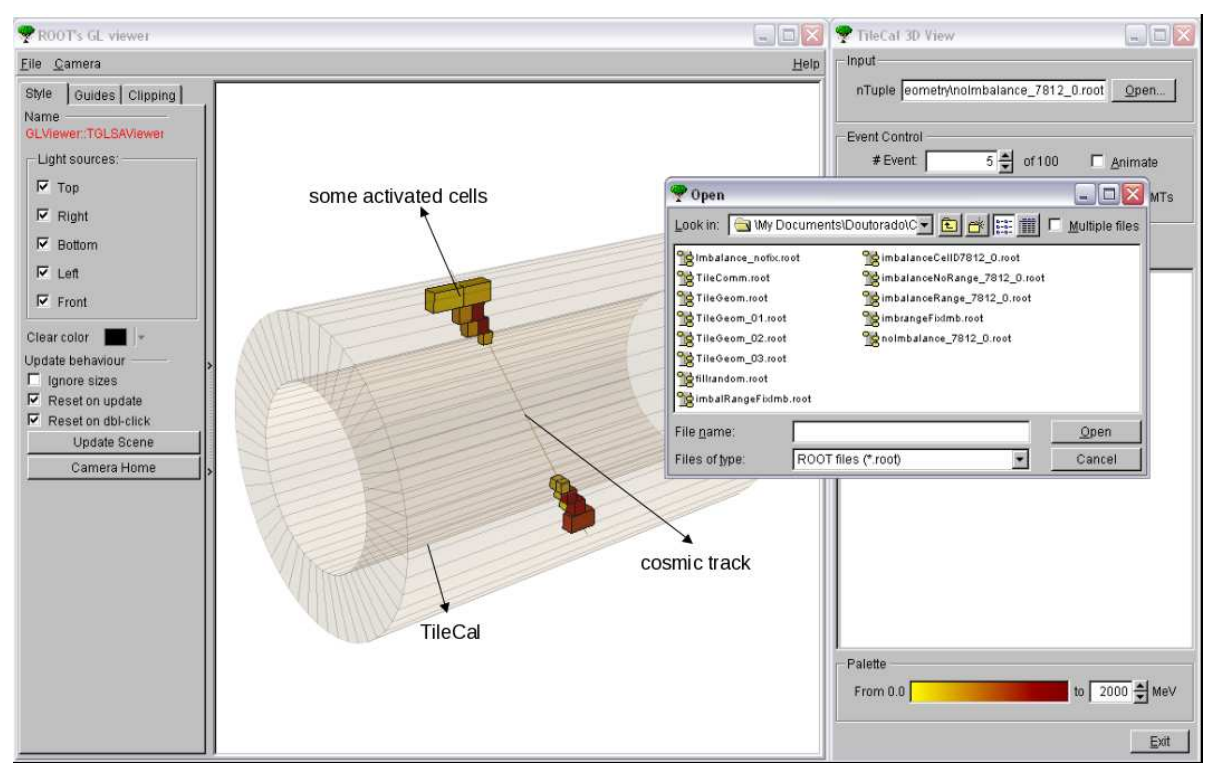

Figure 6: TileCosmicView - Graphical interface.

- Threshold by cell energy - the TileCal has thousands of readout cells; in order to display only the desired cells, a cut value in $\mathrm{MeV}$ can be applied.

- Cell selection with mouse - Desired information for a specific cell are obtained from selecting the cell with the mouse.

- Rotate and zoom - basic traveling commands like rotation, translation and zooming, can be achieved with the mouse; the modifications being seen on the screen instantaneously.

- Show ADC counts - In TileCal specific reconstruct ntuples, information about pulse shape, like ADC counts and fit parameters, are present. The TileCosmicView shows graphics with this informations for the selected cell.

\subsection{The CaloGeoView}

The CaloGeoView is the visualization tool for the combined hadronic and electromagnetic calorimeter ntuples. The controls are pretty much the same as the ones in TileGeoView, but individual TileCal PMT information and pulse shapes are not present there. The threshold and the color palette are independent for the two calorimeters, with green palette for the Electromagnetic and hot palette for the hadronic. Figure 7 presents the CaloGeoView graphical interface where one can identify a level two trigger Region of Interest (RoI) simulation. A simulated cosmic event for the cobined calorimeters can be seen in Figure 8.

\section{Conclusion}

The proposed 3D visualization tools have been extensively used in the commissioning phase of the ATLAS calorimeters. Different kind of problems, either in real data acquisition with cosmics 


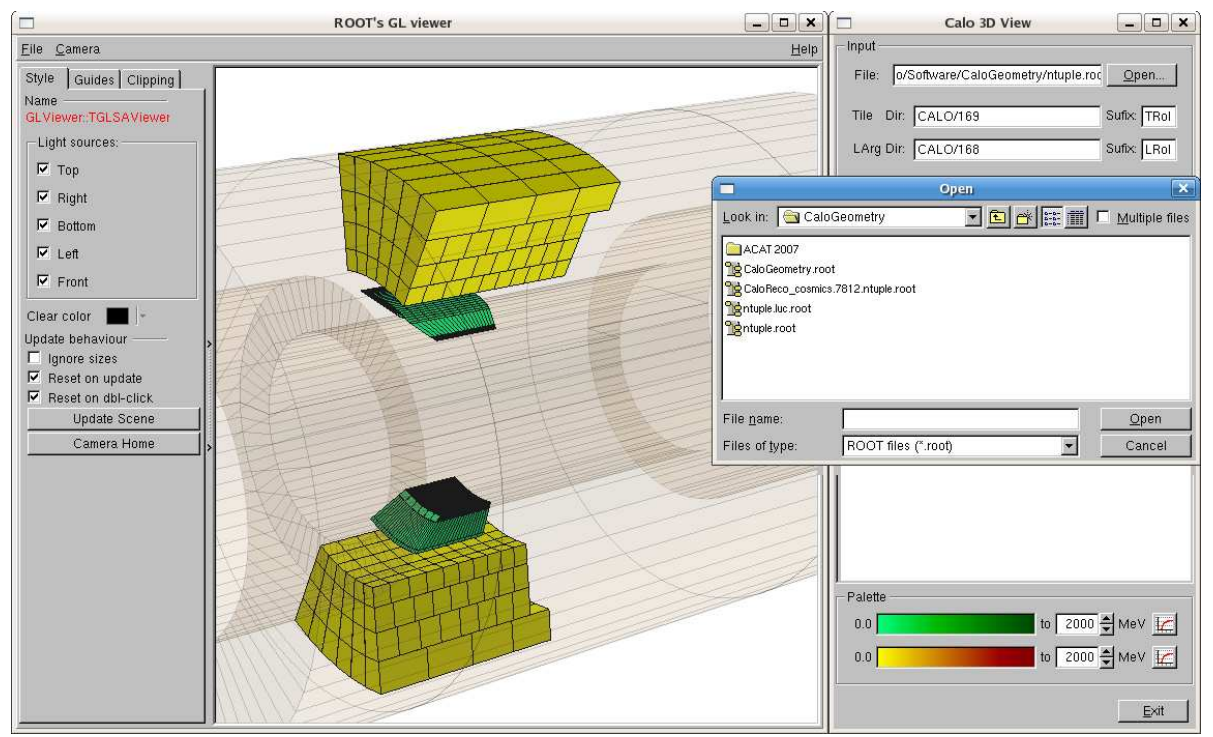

Figure 7: LVL2 RoI seen with CaloGeoView.

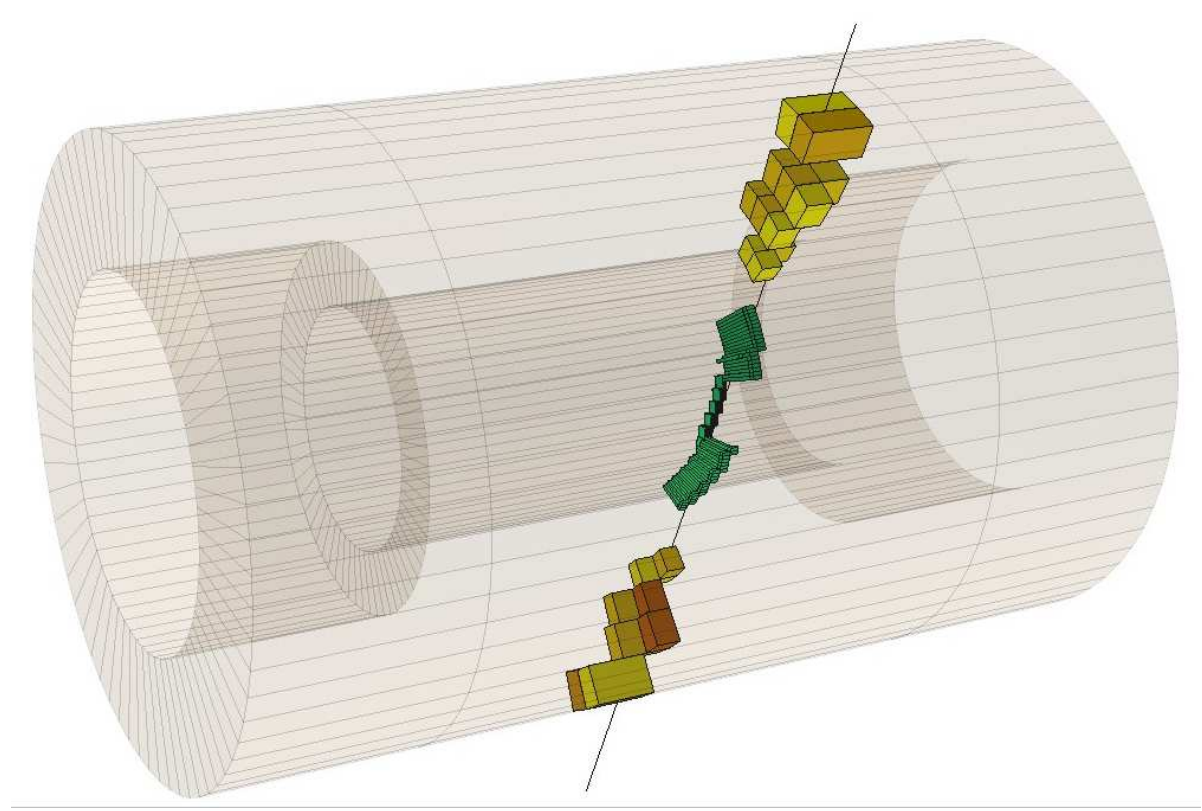

Figure 8: Cosmic simulation seen with CaloGeoView.

or simulated data with LVL2 RoIs, have been discovered thanks to these tools. As next steps, other ATLAS sub-detectors will be implemented in this tool to help in theirs commissioning phase.

The authors would like to thanks CAPES, CNPq and FAPERJ (Brazil) and CERN (Switzerland). We would also like to thank our colleagues from the TileCal / LArg collaboration of the ATLAS detector for providing the simulation data and fruitful discussions concerning this work. 


\section{References}

[1] http://www.atlas.ch

[2] http://atlas.pnpi.nw.ru/INNER.HTM

[3] Calorimetry, ATLAS Technical Proposal. CERN, 1994.

[4] http://atlas.web.cern.ch/Atlas/GROUPS/LIQARGON/Organization/index.html

[5] N.V.Krasnikov, V.A.Matveev (September 1997). Physics at LHC. Physics of Particles and Nuclei 28 (5): 441-470

[6] Overall detector concept, ATLAS Technical Proposal. CERN, 1994.

[7] Introduction and Overview, ATLAS Technical Proposal. CERN, 1994.

[8] https://twiki.cern.ch/twiki/bin/view/Atlas/TriggerDAQ

[9] https://twiki.cern.ch/twiki/bin/view/Atlas/LArCosmicMuonAnalysis

[10] K.Anderson, J.Pilcher, H.Sanders, F.Tang and R.Teuscher. Stand-alone Cosmic Ray Trigger Electronics for the ATLAS Tile Calorimeter. University of Chicago, 2004.

[11] http://root.cern.ch/

[12] ftp://root.cern.ch/root/doc/18Geometry.pdf

[13] http://www.wlap.org/atlas/computing/tutorials/athena/2002/ 